\title{
On an irregular dynamics of certain fragmentation semigroups
}

\author{
Jacek Banasiak
}

Received: 9 August 2010 / Accepted: 8 December 2010 / Published online: 27 January 2011

(C) The Author(s) 2011. This article is published with open access at Springerlink.com

\begin{abstract}
It has been long known that the pure death processes with proliferation can display a chaotic dynamics. In this paper we analyse long time dynamics of fragmentation processes which can be thought of as a generalization of death processes. In particular we show that, if combined with a proliferative process, their dynamics also can become chaotic.
\end{abstract}

Keywords Fragmentation processes - Death processes - Long time behaviour · Compact semigroups $\cdot$ Analytic semigroups $\cdot$ Chaotic semigroups

Mathematics Subject Classification (2000) 47D06

\section{Introduction}

Let us consider an evolution of a denumerable ensemble of objects the state of which is described by an infinite vector $\mathbf{u}=\left(u_{1}, u_{2}, \ldots\right)$. The component $u_{i}$ of $\mathbf{u}$ gives the number of elements of the ensemble which are at a state $i$. For instance, it could be the number of particles consisting of $i$ basic building blocks or the number of cells containing $i$ copies of a particular gene. One of the best understood systems of this kind is the so-called death system. It describes the evolution of an ensemble in which an object in a state $i$ only can move to the preceding state $i-1$ and it is given by

The research was supported by the National Research Foundation of South Africa under grant GUN 2025057 and the University of KwaZulu-Natal Research Fund.

J. Banasiak $(\bowtie)$

School of Mathematical Sciences, University of KwaZulu-Natal,

Durban, South Africa

e-mail: banasiak@ukzn.ac.za

J. Banasiak

Institute of Mathematics, Technical University of Łódź,

Łódź, Poland 


$$
\begin{aligned}
& u_{1}^{\prime}=d_{2} u_{2}, \\
& u_{i}^{\prime}=-d_{i} u_{i}+d_{i+1} u_{i+1}, \quad i \geq 2,
\end{aligned}
$$

where $u_{i}^{\prime}, i=1,2, \ldots$ is the time derivative of $u_{i}$ and the nonnegative sequence $\left(d_{i}\right)_{i \geq 2}$ gives the death rates, that is, the rates of state changes from $i$ to $i-1$. Such systems are formally conservative and there is no annihilation or production of particles in the ensemble as the evolution only consists in a rearrangement of the distribution of the objects of the ensemble among the states. In fact, using e.g. the substochastic semigroup theory [4], one can prove that, irrespective of the growth rate of $d_{i}$, there is a strongly continuous positive stochastic (conservative on positive initial values) semigroup solving (in a suitable sense) Eq. (1) in the space $l^{1}$. Dynamics of this problem is also not particularly interesting. The situation changes, however, if we generalize (1) by including a proliferative process, allowing thus individuals to have an intrinsic growth rate. A typical example would be a cell with $i$ copies of a particular gene producing, upon division, two daughters with the same genotype. In such a case, (1) takes the form

$$
\begin{aligned}
& u_{1}^{\prime}=c_{1} u_{1}+d_{2} u_{2}, \\
& u_{i}^{\prime}=c_{i} u_{i}+d_{i+1} u_{i+1}, \quad i \geq 2,
\end{aligned}
$$

where the sequence $\left(c_{i}\right)_{i \geq 1}$ represents the net growth rate. Here, despite relative simplicity, (2) produces a chaotic dynamics if the death coefficients dominate the growth ones, see $[3,14,16]$.

Further generalizations of the death process are offered by fragmentation processes. Here, a parent particle at a state $n$ can produce a family of daughter particles with states $1 \leq i<n$ in such a way that the sum of the states of all daughter particles must equal $n$. A typical example is offered splitting of a particle into smaller particles, occurring in such a way that the total mass of the ensemble remains unchanged. The system of equations describing such a process is given by

$$
\begin{aligned}
& u_{1}^{\prime}=\sum_{i=2}^{\infty} a_{i} b_{1, i} u_{i} \\
& u_{n}^{\prime}=-a_{n} u_{n}+\sum_{i=n+1}^{\infty} a_{i} b_{n, i} u_{i}, \quad n \geq 2 .
\end{aligned}
$$

As above, the vector $\mathbf{u}=\left(u_{1}, \ldots, u_{n}, \ldots\right)$ describes the distribution of numbers of particles with respect to their size (or mass). The sequence $\left(a_{n}\right)_{n \geq 1}$, with $a_{1}=0$, defines the fragmentation rates, that is, the rates at which the particles of mass $n$ undergo splitting. The matrix $\left(b_{i, n}\right)_{1 \leq i<n, 2 \geq n}$ gives the distribution of the numbers of size $i$ particles resulting from splitting of a size $n$ parent. Following the introduction of the model, we assume that $a_{n}, b_{i, n}$ $n \geq 2,1 \leq i<n$, are nonnegative numbers with

$$
\sum_{i=1}^{n-1} i b_{i, n}=n
$$

This equation expresses the conservation of mass in each fragmentation event, described above, and plays a crucial role in its analysis. 
There are two natural spaces in which (3) could be analysed. Following the death problem, we could consider (3) in the space $l^{1}$ in which the norm of a nonnegative element $\mathbf{u}$,

$$
\|\mathbf{u}\|_{0}=\sum_{i=1}^{\infty} u_{i}
$$

gives the total number of particles in the ensemble. A quick reflection, however, warns that, contrary to the death system, in fragmentation the number of particles grows and we loose the advantage of the conservativeness of the process. Indeed, as we shall see later in Corollary 1(i), (4) in general does not generate a strongly continuous semigroup in $l^{1}$ unless the coefficients $a_{n}$ are bounded. On the other hand, (4) shows that the fragmentation process is just a rearrangement of mass among particles and thus the total mass of the ensemble should not change. Hence, fragmentation should be conservative in the space $l_{1}^{1}$ of sequences with the weighted $l^{1}$ norm

$$
\|\mathbf{u}\|_{1}=\sum_{i=1}^{\infty} i u_{i}
$$

which, for a nonnegative $\mathbf{u}$, gives the mass of the ensemble. In fact, in this space the analysis of (3) proves to be quite straightforward, [4]. We begin with the diagonal operator $A$ which is the realization of the multiplication

$$
\mathcal{A} \mathbf{u}=\left\{0, a_{2} u_{2}, a_{3} u_{3} \ldots\right\}
$$

on the domain $D(A)=\left\{\mathbf{u} \in l_{1}^{1} ; \mathcal{A} \mathbf{u} \in l_{1}^{1}\right\}$. By $\mathcal{B}$ we denote the off-diagonal expression

$$
\mathcal{B} \mathbf{u}=\left(\sum_{i=n+1}^{\infty} a_{i} b_{n, i} u_{i}\right)_{n \geq 1},
$$

defined whenever each series converges. To define the associated operator $B$ we observe that $\mathcal{B} \mathbf{u} \in l_{1}^{1}$ for $\mathbf{u} \in D(A)$. Hence, we can define $B=\left.\mathcal{B}\right|_{D(A)}$ and, again for $\mathbf{u} \in D(A)$,

$$
\sum_{i=1}^{\infty} i((-A+B) \mathbf{u})_{i}=-\sum_{i=2}^{\infty} i a_{i} u_{i}+\sum_{n=1}^{\infty} n\left(\sum_{i=n+1}^{\infty} a_{i} b_{n, i} u_{i}\right)=0 .
$$

This allows for an application of the substochastic semigroup theory, [4], asserting existence of an extension $(F, D(F))$ of $(-A+B, D(A))$ which generates a positive contractive semigroup, say $(S(t))_{t \geq 0}$. Furthermore, in a standard way, as in [4, Sects. 6.3, 7.4], we obtain that $F=\overline{-A+B}$.

It is easy to see that the $N$-dimensional subspace $X_{N}$ of $X_{1}$, consisting of vectors $\mathbf{u}=$ $\left(u_{1}, \ldots, u_{N}, 0 \ldots,\right)$ is invariant under the action of the fragmentation semigroup $(S(t))_{t \geq 0}$, see also [4, Proposition 8.8]. Hence, by induction, $(S(t))_{t \geq 0}$ is given by a matrix of the form

$$
\mathcal{S}(t)=\left(\begin{array}{cccccc}
1 & s_{1,2}(t) & \ldots & s_{1, n}(t) & s_{1, n+1}(t) & \ldots \\
0 & e^{-a_{2} t} & \ldots & s_{2, n}(t) & s_{2, n+1}(t) & \ldots \\
\vdots & \vdots & \vdots & \vdots & \vdots & \vdots \\
0 & 0 & \ldots & e^{-a_{n} t} & s_{n, n+1}(t) & \ldots \\
\vdots & \vdots & \vdots & \vdots & \vdots & \vdots
\end{array}\right)
$$

where the entries $s_{k, n}(t), k \geq 1, n \geq k+1$, can be found by the following lemma. 
Lemma 1 Denote $s_{1,1}(t)=1$ and $s_{k, k}(t)=e^{-a_{k} t}, k \geq 1$. Then for any $k \geq 1$ and $n \geq k+1$

$$
s_{k, n}(t)=a_{n} e^{-a_{n} t} \sum_{i=k}^{n-1} b_{i, n} \int_{0}^{t} s_{k, i}(\tau) e^{a_{n} \tau} d \tau .
$$

Proof Let us consider $\mathbf{f}=\left(\delta_{i n}\right)_{i \in \mathbb{N}}$. Then, for any $k \leq n$,

$$
\mathbf{u}(t)=S(t) \mathbf{f}=\left(s_{1, n}(t), s_{2, n}(t), \ldots, s_{n, n}(t), 0, \ldots\right) .
$$

On the other hand, $\mathbf{u}(t)$ can be expressed in a different way. We know that $u_{n}(t)=s_{n, n}(t)=$ $e^{-a_{n} t}$ so that we can replace the unknown $u_{n}(t)$ and consider the $n-1 \times n-1$ inhomogeneous system

$$
u_{k}^{\prime}(t)=-a_{k} u_{k}+\sum_{i=k+1}^{n-1} a_{i} b_{k, i} u_{i}(t)+a_{n} b_{k, n} e^{-a_{n} t}, \quad 1 \leq k \leq n-1,
$$

(recall $a_{1}=0$ ) solution of which can be found by the Duhamel formula. Combining this with (12) we get

$$
s_{k, n}(t)=a_{n} e^{-a_{n} t} \sum_{i=k}^{n-1} b_{i, n} \int_{0}^{t} s_{k, i}(t-\sigma) e^{a_{n}(t-\sigma)} d \sigma,
$$

which, using $\tau=t-\sigma$, gives (11) for any $k \leq n-1$. Since $n$ was arbitrary, (11) is proved.

\section{Decay to the steady state}

Let us denote $\mathbf{e}_{1}=(1,0,0, \ldots)$ and let $M(\stackrel{\circ}{\mathbf{u}})=\sum_{i=1}^{\infty} i \stackrel{\circ}{i}_{i}$ be the initial mass of the ensemble. From the physics of the problem it is obvious that any solution should converge to the stationary solution in which the whole mass is concentrated in monomers, that is, to $M\left(\mathbf{u}_{)} \mathbf{e}_{1}\right.$. The proof of this fact given in [9] is technically quite involved. Here we present an elementary alternative proof of this fact, based on the substochastic semigroup theory and explore some of its consequences.

Together with (3) we consider the equation for $\mathbf{u}^{[2]}=\left(u_{2}, u_{3}, \ldots\right)$ :

$$
u_{n}^{\prime}=-a_{n} u_{n}+\sum_{i=n+1}^{\infty} a_{i} b_{n, i} u_{i}, \quad n \geq 2,
$$

and the corresponding matrix of coefficient we denote by $\mathcal{F}^{[2]}$. Further, we denote by $\mathcal{A}^{[2]}$ the diagonal part of $\mathcal{F}^{[2]}: \mathcal{A}^{[2]} \mathbf{v}=\left(a_{2} v_{2}, a_{3} v_{3}, \ldots\right)$ and by $\mathcal{B}^{[2]}$ the off-diagonal part $\mathcal{B}^{[2]}=$ $\mathcal{F}^{[2]}+\mathcal{A}^{[2]}$. Let

$$
X^{[2]}=\left\{\mathbf{v} ; \sum_{i=2}^{\infty} i\left|v_{i}\right|<\infty\right\} .
$$

On $X^{[2]}$ we can define the operator $A^{[2]}=\left.\mathcal{A}^{[2]}\right|_{D\left(A^{[2]}\right)}$ with

$$
D\left(A^{[2]}\right)=\left\{\mathbf{v} \in l_{1}^{1} ; \sum_{i=2}^{\infty} i a_{i}\left|v_{i}\right|<\infty\right\} .
$$


In what follows, for any subspace $Z$ of $l_{1}^{1}$ ( or $l^{1}$ ), by $Z_{+}$we denote the cone of nonnegative elements of $Z$. Then, as for the full fragmentation operator, we have for $\mathbf{v} \in D\left(A^{[2]}\right)_{+}$

$$
\begin{aligned}
0 & <\sum_{i=2}^{\infty}\left(\sum_{n=i+1}^{\infty} i a_{n} b_{i, n} v_{n}\right)=\sum_{n=3}^{\infty} a_{n} v_{n}\left(\sum_{i=2}^{n-1} i b_{i, n}\right)=\sum_{n=3}^{\infty} a_{n} v_{n}\left(n-b_{1, n}\right) \\
& \leq \sum_{n=3}^{\infty} a_{n} v_{n} n<+\infty
\end{aligned}
$$

hence $B^{[2]}=\left.\mathcal{B}^{[2]}\right|_{D\left(A^{[2]}\right)}$ is well defined and we have

$$
\sum_{n=2}^{\infty} n\left(\left(A^{[2]}+B^{[2]}\right) \mathbf{v}\right)_{n}=-\sum_{n=2}^{\infty} a_{n} b_{1, n} v_{n}=:-c(\mathbf{v}), \quad \mathbf{v} \in D\left(A^{[2]}\right)_{+} \cdot
$$

Thus we can use the substochastic semigroup theory. Standard calculation using the Arlotti extension, see [4, Chapter 7], gives

$$
\begin{aligned}
\sum_{n=2}^{N}\left(-n a_{n} v_{n}+\sum_{i=n+1}^{\infty} n a_{i} b_{n, i} v_{i}\right)= & -\sum_{n=2}^{N} a_{n} b_{1, n} v_{n}+a_{N+1} v_{N+1} \sum_{n=2}^{N} n b_{n, N+1} \\
& +\sum_{i=N+2}^{\infty} a_{i} v_{i} \sum_{n=2}^{N} n b_{n, i},
\end{aligned}
$$

hence

$$
\lim _{N \rightarrow \infty} \sum_{n=2}^{N}\left(-n a_{n} v_{n}+\sum_{i=n+1}^{\infty} n a_{i} b_{n, i} v_{i}\right) \geq-c(\mathbf{v})
$$

for appropriate $\mathbf{v}$ as specified in [4, Theorem 6.22]. Thus the closure $F^{[2]}=\overline{-A^{[2]}+B^{[2]}}$ generates a substochastic semigroup $\left(S^{[2]}(t)\right)_{t \geq 0}$ in $X^{[2]}$ satisfying, for $\left.\stackrel{\circ}{\mathbf{v}} \in D_{\left(F^{[2]}\right.}\right)_{+}$,

$$
\frac{d M^{[2]}}{d t}=-c\left(S^{[2]}(t) \stackrel{\circ}{\mathbf{v}}\right)
$$

By integrating and extending by density, as in [4, Proposition 6.9], we further obtain, for $\stackrel{\circ}{\mathbf{v}} \in X_{+}^{[2]}$,

$$
M^{[2]}(t)=M^{[2]}(0)-\int_{0}^{t} c\left(S^{[2]}(s) \stackrel{\circ}{\mathbf{v}}\right) d s,
$$

where $M^{[2]}(t)=\sum_{n=2}^{\infty} n\left(S^{[2]}(t) \stackrel{\circ}{\mathbf{v}}\right)_{n}$.

Let $X_{N}^{[2]}$ be the space of finite sequences $\left(v_{2}, \ldots, v_{N}\right)$ equipped with the norm induced from $X^{[2]}$; we identify it with the closed subspace of $X_{N}^{[2]}$ consisting of sequences of the form $\left(v_{2}, v_{3}, \ldots, v_{N}, 0,0, \ldots\right)$. It is clear that $X_{N}^{[2]}$ is invariant under the action of $\left(S^{[2]}(t)\right)_{t \geq 0}$ and that the finite-dimensional semigroup $\left(S_{N}^{[2]}(t)\right)_{t \geq 0}$ generated by the restriction of $\mathcal{F}^{[2]}$ to $X_{N}^{[2]}$ satisfies

$$
\tilde{S}_{N}^{[2]}(t) \stackrel{\circ}{\mathbf{v}}=S^{[2]}(t) \stackrel{\circ}{\mathbf{v}}, \quad \stackrel{\circ}{\mathbf{v}} \in X_{N}^{[2]}
$$


where by $\tilde{S}_{N}^{[2]}(t)$ we defined the extension of $S_{N}(t)$ by zero to $X^{[2]}$. This fact yields the representation of $\left(S^{[2]}(t)\right)_{t \geq 0}$ as

$$
S^{[2]}(t)=\left(\begin{array}{ccccc}
e^{-a_{2} t} & s_{2,3}(t) & \ldots & s_{2, n+1}(t) & \ldots \\
\vdots & \vdots & \vdots & \vdots & \vdots \\
0 & \ldots & e^{-a_{n} t} & s_{n, n+1}(t) & \ldots \\
\vdots & \vdots & \vdots & \vdots & \vdots
\end{array}\right),
$$

where the entries are the same as in (10) as can be established by iteratively solving (14) and (3) with the initial condition $\stackrel{\circ}{\mathbf{v}}=\left(\stackrel{\circ}{v}_{2}, \stackrel{\circ}{v}_{3}, \ldots, \stackrel{\circ}{v}_{N}\right)$ and arbitrary but finite $N$.

A crucial observation is that for an arbitrary nonnegative $\stackrel{\circ}{\mathbf{v}} \in X^{[2]}$ we have

$$
\lim _{N \rightarrow \infty} \tilde{S}_{N}(t) \stackrel{\circ}{\mathbf{v}}=S^{[2]}(t) \stackrel{\circ}{\mathbf{v}},
$$

where $P_{N} \stackrel{\circ}{\mathbf{v}}=\left(\stackrel{\circ}{v}_{2}, \stackrel{\circ}{v}_{3}, \ldots, \stackrel{\circ}{v_{N}}\right)$, monotonically with respect to $N$ for any $t$, and uniformly in $t$ on bounded time intervals in the norm of $X^{[2]}$ (the proof is as in [4, Proposition 8.8]). Now, since $c$ is a non-negative functional, by the Lebesgue monotonic convergence theorem (for series) we have

$$
\int_{0}^{t} c\left(S^{[2]}(\tau) \stackrel{\circ}{\mathbf{v}}\right) d \tau=\lim _{N \rightarrow \infty} \int_{0}^{t} c\left(\tilde{S}_{N}(\tau) P_{N} \stackrel{\circ}{\mathbf{v}}\right) d \tau=\lim _{N \rightarrow \infty} \int_{0}^{t} \sum_{n=2}^{N} a_{n} b_{1, n} \sum_{i=n}^{N} s_{n i}(\tau) v_{i} d \tau .
$$

We start with a preliminary observation.

Lemma 2 Let $\alpha \geq 0$ and $\stackrel{\circ}{\mathbf{u}} \in l_{1}^{1}$ be fixed and let $\stackrel{\circ}{\mathbf{u}}^{[2]}:=\left(\stackrel{\circ}{u}_{2}, \stackrel{\circ}{u}_{3}, \ldots\right)$. Then the following are equivalent

$$
\begin{gathered}
\lim _{t \rightarrow \infty} e^{\alpha t}\left\|S(t) \stackrel{\circ}{\mathbf{u}}-M(\stackrel{\circ}{\mathbf{u}}) \mathbf{e}_{1}\right\|_{1}=0, \\
\lim _{t \rightarrow \infty} e^{\alpha t} S^{[2]}(t) \stackrel{\circ}{\mathbf{u}^{[2]}}=0 .
\end{gathered}
$$

Proof Denote $M_{1}(t)=\sum_{k=1}^{\infty} s_{1 k}(t) \stackrel{\circ}{u_{k}}$ and $M=M(\stackrel{\circ}{\mathbf{u}})=M_{1}(0)$, for a given $\stackrel{\circ}{\mathbf{u}} \geq 0$. First assume that (18) holds. We have

$$
\begin{aligned}
& \left\|S(t) \stackrel{\circ}{\mathbf{u}}-M \mathbf{e}_{1}\right\|_{1}=\left|\sum_{k=1}^{\infty} s_{1 k}(t) \stackrel{\circ}{\stackrel{u}{u}_{k}}-M\right|+\left|\sum_{l=2}^{\infty} l\left(\sum_{k=l}^{\infty} s_{l k}(t) \stackrel{\circ}{u_{k}}\right)\right| \\
& =\left|M_{1}(t)-M\right|+\left\|S^{[2]}(t) \stackrel{\circ}{[2]}^{[2]}\right\|_{X^{[2]}} \text {, }
\end{aligned}
$$

hence an exponential decay to 0 of the left hand side implies the same for each term on the right hand side and, in particular, the decay of $\left(S^{[2]}(t)\right)_{t \geq 0}$ to zero at the same rate.

To prove the result in the opposite direction, we note that

$$
S(t) \stackrel{\circ}{\mathbf{u}}=\mathbf{e}_{1} \sum_{k=1}^{\infty} s_{1 k}(t) \stackrel{\circ}{u}_{k}+S^{[2]}(t) \stackrel{\circ}{\mathbf{u}}^{[2]}
$$

so that

$$
e^{\alpha t} S(t) \stackrel{\circ}{\mathbf{u}}-e^{\alpha t} M_{1}(t) \mathbf{e}_{1}=e^{\alpha t} S^{[2]}(t) \stackrel{\circ}{\mathbf{u}^{[2]}} \rightarrow 0
$$


as $t \rightarrow 0$. Using the reverse triangle inequality, we get

$$
\begin{aligned}
0 & \leftarrow\left\|e^{\alpha t} S(t) \stackrel{\circ}{\mathbf{u}}-e^{\alpha t} M_{1}(t) \mathbf{e}_{1}\right\|_{1} \geq\left|\left\|e^{\alpha t} S(t) \mathbf{u}\right\|_{1}-\left\|e^{\alpha t} M_{1}(t) \mathbf{e}_{1}\right\|_{1}\right| \\
& =e^{\alpha t}\left|M-M_{1}(t)\right|,
\end{aligned}
$$

so (18) follows by (20).

Now we can prove the main result of this section.

Theorem 1 We have

$$
\lim _{t \rightarrow \infty}\left\|S(t) \stackrel{\circ}{\mathbf{u}}-M(\stackrel{\circ}{\mathbf{u}}) \mathbf{e}_{1}\right\|_{1}=0
$$

for any $\stackrel{\circ}{\mathbf{u}} \in l_{1,+}^{1}\left(\right.$ and also for $\left.l_{1}^{1}\right)$ if an only if $a_{n} \neq 0$ for all $n \geq 2$.

Proof Let us consider $\stackrel{\circ}{\mathbf{u}} \in X_{+}^{[2]}$ and $\stackrel{\circ}{\mathbf{u}}_{N}=P_{N} \stackrel{\circ}{\mathbf{u}}$. Then $\mathbf{u}_{N}=\tilde{S}^{[2]}(t) \stackrel{\circ}{\mathbf{u}}_{N}$ is the (extended by zero) solution of the truncated equation. Denote

$$
M_{N}^{[2]}(t)=\sum_{k=2}^{N} k\left(\tilde{S}^{[2]}(t) \stackrel{\circ}{\mathbf{u}_{N}}\right)_{k}
$$

Using the fact that the truncated system defining $\left(S_{N}^{[2]}(t)\right)_{t \geq 0}$ is finite dimensional and all its eigenvalues are negative, we have

$$
\lim _{t \rightarrow \infty} M_{N}^{[2]}(t)=0
$$

On the other hand,

$$
M_{N}^{[2]}(t)=M_{N}^{[2]}(0)-\int_{0}^{t} c\left(\tilde{S}^{[2]}(s) \stackrel{\circ}{\mathbf{u}_{N}}\right) d s
$$

hence

$$
\lim _{t \rightarrow \infty} \int_{0}^{t} c\left(\tilde{S}^{[2]}(s) \stackrel{\circ}{\mathbf{u}_{N}}\right) d s=M_{N}^{[2]}(0) .
$$

Since $\mathbf{u}_{N}(t)$ monotonically increases to $\mathbf{u}(t)$ and $c$ is nonnegative, from (16) we obtain

$$
M^{[2]}(t)=M^{[2]}(0)-\int_{0}^{t} c\left(S^{[2]}(s) \stackrel{\circ}{\mathbf{u}}\right) d s \leq M^{[2]}(0)-\int_{0}^{t} c\left(\tilde{S}^{[2]}(s) \stackrel{\circ}{\mathbf{u}_{N}}\right) d s .
$$

Now, for any $\epsilon$ we can find $N$ such that $\left|M^{[2]}(0)-M_{2, N}(0)\right| \leq \epsilon$ and thus we can write

$$
0 \leq \lim _{t \rightarrow \infty} M^{[2]}(t) \leq \epsilon+\lim _{t \rightarrow \infty}\left|M_{N}^{[2]}(0)-\int_{0}^{t} c\left(S^{[2]}(s) \stackrel{\circ}{\mathbf{u}}_{N}\right) d s\right|=\epsilon .
$$

Since $\epsilon>0$ is arbitrary, we have

$$
\lim _{t \rightarrow \infty} M^{[2]}(t)=0 .
$$

The result follows now from Lemma 2 with $\alpha=0$. 
On the other hand, if $a_{i}=0$ for some $i \geq 2$, then the whole $i$ th column in $\mathcal{F}$ is zero. If we take the initial condition $\stackrel{\circ}{\mathbf{u}}=\left(\delta_{N j}\right)_{j \in \mathbb{N}}$ then, as argued above, $S(t) \stackrel{\circ}{\mathbf{u}}$ can be identified with $S_{N}(t) \stackrel{\circ}{\mathbf{u}}$. But then $u_{N}(t)=1$ for all $t$ and thus the we cannot have convergence to $\mathbf{e}_{1}$.

\section{Non-analytic and non-compact fragmentation semigroup with an unbounded generator}

A natural question is whether the result of Theorem 1 can be improved to an exponential convergence, that is, whether it is possible to prove that (18) holds with some $\alpha<0$. We note that in many applications semigroups satisfying (18) with $\alpha<0$ are said to to have asynchronous exponential growth (AEG) property. The name refers to the fact that the evolution of the population very quickly becomes independent of a particular initial condition. In [8] we characterized a class of fragmentation problems having the AEG property. Namely, we proved the following theorem.

Theorem 2 If the fragmentation semigroup $(S(t))_{t \geq 0}$ is compact, then it has asynchronous exponential growth property; that is, there is $\alpha>0$ such that for any $\stackrel{\circ}{\mathbf{u}}$ there $i$ is $K$ such that

$$
\left\|S(t) \stackrel{\circ}{\mathbf{u}}-M(\stackrel{\circ}{\mathbf{u}}) \mathbf{e}_{1}\right\| \leq K e^{-\alpha t} .
$$

In particular, if there are positive constants $k_{0}, C, \delta$ and a positive sequence $\left(\phi_{k}\right)_{k \in \mathbb{N}}$ such that for $k>k_{0} a_{k} \geq k^{\delta}, \phi_{k} \geq k^{2}$ and

$$
\phi_{k} b_{k, n} \leq C \sum_{i=1}^{k} i b_{i, n}
$$

then the semigroup $(S(t))_{t \geq 0}$ is compact and analytic.

We note that this result is not a simple corollary of the classical result on AEG, see e.g. [6, Theorem 51], [10, Theorem 8.17] or [13, Theorem VI.3.5], as fragmentation semigroups are not irreducible.

It follows that the assumptions on the fragmentation coefficients introduced in Theorem 2 mean that the process has a tendency to create particles of smaller sizes. Thus the result seems to be intuitive as the concentration of mass in small particles makes the process close to finite dimensional. The question is whether the introduced assumptions are only technical or whether they reflect a real feature of the process. While we are not able to give sufficient and necessary conditions for analyticity and compactness of fragmentation semigroups in the general case, we can provide examples showing that the above physical intuition is correct, that is, that fragmentation processes in which a substantial number of the daughter particles has large masses, do not generate compact analytic semigroups. For this purpose, we consider fragmentation processes in which only a single particle can detach from a size $n$ parent, creating a pair consisting of a monomer and an $n-1$-mer, as in the Becker-Döring model, see [2] and references therein. This is modelled by

$$
b_{1,2}=2, \quad b_{1, n}=b_{n-1, n}=1, \quad b_{k, n}=0 \text { for } 1<k<n-1, k \geq n \text { and } n \geq 2 .
$$

In such a case, condition (23) for $k=n-1$ reads

$$
(n-1)^{2} b_{n-1, n}=(n-1)^{2} \leq C \sum_{i=1}^{n-1} i b_{i, n}=C(1+(n-1))=C n
$$


which is obviously false. Therefore such $b_{k, n}$ s do not satisfy (23) with $\phi_{k} \geq k^{2}$ which was required to prove the generation of an analytic semigroup. We will consider two fragmentation processes with such coefficients $b_{k, n}$, one with unbounded and one with bounded fragmentation rates. As we shall see, despite some similarities, they have different long time asymptotics.

3.1 Fragmentation with unbounded fragmentation rates

System (3) with $a_{n}=n$ for $n \geq 2, a_{1}=0$ and $\left(b_{k, n}\right)_{1 \leq k<n, n \geq 2}$ satisfying (24) takes the form

$$
\begin{aligned}
& u_{1}^{\prime}=4 u_{2}+\sum_{n=3}^{\infty} n u_{n}, \\
& u_{i}^{\prime}=-i u_{i}+(i+1) u_{i+1}, \quad i=2, \ldots
\end{aligned}
$$

We have the following result.

Theorem 3 The semigroup $(S(t))_{t \geq 0}$ associated with the system (25) is neither analytic nor compact.

Proof We apply Lemma 1 to find an explicit expression of the semigroup. First let $i>1$. Then $s_{i i}(t)=e^{-i t}$ and

$$
s_{i, i+1}(t)=(i+1) e^{-(i+1) t} \int_{0}^{t} e^{\tau} d \tau=(i+1) e^{-i t}\left(1-e^{-t}\right) .
$$

Making induction ansatz

$$
s_{i, i+k}(t)=\left(\begin{array}{c}
i+k \\
k
\end{array}\right) e^{-(i+k) t}\left(e^{t}-1\right)^{k}
$$

we evaluate

$$
\begin{aligned}
s_{i, i+k+1}(t) & =(i+k+1)\left(\begin{array}{c}
i+k \\
k
\end{array}\right) e^{-(i+k+1) t} \int_{0}^{t}\left(e^{\tau}-1\right)^{k} e^{\tau} d \tau \\
& =\left(\begin{array}{c}
i+k+1 \\
k+1
\end{array}\right) e^{-(i+k+1) t}\left(e^{t}-1\right)^{k+1},
\end{aligned}
$$

so that (26) is proven. For $i=1$, we have $s_{11}(t)=1$,

$$
s_{1,2}=a_{2} e^{-a_{2} t} b_{1,2} \int_{0}^{t} s_{11}(\tau) e^{a_{2} \tau} d \tau=4 e^{-2 t} \int_{0}^{t} e^{2 \tau} d \tau=2\left(1-e^{-2 t}\right)
$$

and

$$
s_{1,3}(t)=3 e^{-3 t}\left(\int_{0}^{t} e^{3 \tau} d \tau+2 \int_{0}^{t}\left(1-e^{-2 \tau}\right) e^{3 \tau} d \tau\right)=3\left(1-e^{-t}+e^{-t}\left(1-e^{-t}\right)^{2}\right) .
$$

This justifies the ansatz

$$
s_{1, n}(t)=n\left(1-e^{-t}+e^{-t}\left(1-e^{-t}\right)^{n-1}\right) .
$$


Evaluating $s_{1, n+1}$, we get

$$
\begin{aligned}
& s_{1, n+1}(t)=(n+1) e^{-(n+1) t}\left(\int_{0}^{t} e^{(n+1) \tau} d \tau+n \int_{0}^{t}\left(1-e^{\tau}+e^{-\tau}\left(1-e^{-\tau}\right)^{n-1}\right) e^{(n+1) \tau} d \tau\right) \\
& =(n+1)\left(1-e^{-t}+e^{-t}\left(1-e^{-t}\right)^{n}\right)
\end{aligned}
$$

and (27) is proved.

To prove that $(S(t))_{t \geq 0}$ is not analytic, we slightly adapt the argument used in [15, Appendix A] for the pure death problem. Indeed, if $(S(t))_{t \geq 0}$ were analytic then, in particular, for any $\mathbf{x} \in l_{1}^{1}, t \rightarrow S(t) \mathbf{x}$ would be differentiable and thus $S^{\prime}(t)=F S(t)$ would be a bounded operator for each $t>0$. Since, for $\mathbf{e}_{n}=\left(\delta_{i n}\right)_{i \geq 1}, S(t) \mathbf{e}_{n}=$ $\left(s_{1 n}(t), s_{2 n}(t), \ldots, s_{n n}(t), 0, \ldots\right)$, we have

$$
S^{\prime}(t)=\left\{s_{i j}^{\prime}(t)\right\}_{1 \leq i, j \leq \infty} .
$$

For the entries on the right hand side, we have $s_{11}^{\prime}(t)=0$,

$$
s_{1 n}^{\prime}(t)=n e^{-t}\left(1+\left(n e^{-t}-1\right)\left(1-e^{-t}\right)^{n-2}\right),
$$

and

$$
s_{i n}^{\prime}(t)= \begin{cases}0 & \text { for } n<i \\
\left(\begin{array}{l}
n \\
i
\end{array}\right) e^{-i t}\left(1-e^{-t}\right)^{n-i-1}\left(n e^{-t}-i\right) & \text { for } n \geq i\end{cases}
$$

Now

$$
\left\|S^{\prime}(t) \mathbf{e}_{n}\right\|_{1}=\sum_{i=1}^{n} i\left|s_{i n}^{\prime}(t)\right| \geq \sum_{i=2}^{n} i\left|s_{i n}^{\prime}(t)\right|=\sum_{i=2}^{n} i\left(\begin{array}{l}
n \\
i
\end{array}\right) e^{-i t}\left(1-e^{-t}\right)^{n-i-1}\left|n e^{-t}-i\right| .
$$

Following [15], we take a particular time $\bar{t}=\ln 2$ so that $e^{-\bar{t}}=1 / 2$ and

$$
\begin{aligned}
\left\|S^{\prime}(t) \mathbf{e}_{2 n}\right\|_{1} & \geq 2 \sum_{i=2}^{2 n} i\left(\begin{array}{c}
2 n \\
i
\end{array}\right) 2^{-2 n}|n-i| \\
& \geq 2^{-2 n+1}\left(\sum_{i=2}^{n-1} i\left(\begin{array}{c}
2 n \\
i
\end{array}\right)(n-i)+\sum_{i=n+1}^{2 n-2} i\left(\begin{array}{c}
2 n \\
i
\end{array}\right)(i-n)\right) \\
& =2^{-2 n+1} 2 n \sum_{i=2}^{n-1}\left(\begin{array}{c}
2 n \\
i
\end{array}\right)(n-i),
\end{aligned}
$$

where we changed variables according to $i=2 n-k$ and used $\left(\begin{array}{c}2 n \\ 2 n-k\end{array}\right)=\left(\begin{array}{c}2 n \\ k\end{array}\right)$. Next we have

$$
\sum_{i=2}^{n-1}\left(\begin{array}{c}
2 n \\
i
\end{array}\right)=\sum_{i=0}^{n-1}\left(\begin{array}{c}
2 n \\
i
\end{array}\right)-1-2 n=\frac{1}{2} 2^{2 n}-\frac{1}{2}\left(\begin{array}{c}
2 n \\
n
\end{array}\right)-1-2 n .
$$

Similarly

$$
\sum_{i=2}^{n-1} i\left(\begin{array}{c}
2 n \\
i
\end{array}\right)=\sum_{i=1}^{n-1} i\left(\begin{array}{c}
2 n \\
i
\end{array}\right)-2 n=2 n \sum_{i=0}^{n-2}\left(\begin{array}{c}
2 n-1 \\
i
\end{array}\right)-2 n
$$


Now, using $i=2 n-1-k$,

$$
2^{2 n-1}=2 \sum_{i=0}^{n-2}\left(\begin{array}{c}
2 n-1 \\
i
\end{array}\right)+\left(\begin{array}{c}
2 n-1 \\
n-1
\end{array}\right)+\left(\begin{array}{c}
2 n-1 \\
n
\end{array}\right) .
$$

However,

$$
\left(\begin{array}{c}
2 n-1 \\
n-1
\end{array}\right)+\left(\begin{array}{c}
2 n-1 \\
n
\end{array}\right)=2\left(\begin{array}{c}
2 n-1 \\
n
\end{array}\right)=\left(\begin{array}{c}
2 n \\
n
\end{array}\right)
$$

and thus

$$
\sum_{i=2}^{n-1} i\left(\begin{array}{c}
2 n \\
i
\end{array}\right)=n\left(2^{2 n-1}-\left(\begin{array}{c}
2 n \\
n
\end{array}\right)-2\right)
$$

Hence

$$
\left\|S^{\prime}(\bar{t}) \mathbf{e}_{2 n}\right\|_{1} \geq 2^{-2 n+1} 2 n \sum_{i=2}^{n-1}\left(\begin{array}{c}
2 n \\
i
\end{array}\right)(n-i)=2^{-2 n+1} 2 n\left(\frac{n}{2}\left(\begin{array}{c}
2 n \\
n
\end{array}\right)+n-2 n^{2}\right) .
$$

Using [1, Formula 6.1.38]

$$
n !=\sqrt{2 \pi} n^{n+1 / 2} e^{-n+\theta / 12 n}, \quad 0<\theta<1
$$

we find that

$$
2^{-2 n}\left(\begin{array}{c}
2 n \\
n
\end{array}\right)=\frac{\gamma_{n}}{\sqrt{\pi n}}
$$

where $\gamma_{n} \rightarrow 1$ as $n \rightarrow \infty$. Taking into account that $\left\|\mathbf{e}_{2 n}\right\|_{1}=2 n$ we find

$$
\frac{\left\|S^{\prime}(\bar{t}) \mathbf{e}_{2 n}\right\|_{1}}{\left\|\mathbf{e}_{2 n}\right\|_{1}} \geq \frac{\sqrt{n} \gamma_{n}}{\sqrt{\pi}}-4 n^{2} 2^{-2 n}
$$

which shows that $S^{\prime}(\bar{t})$ is unbounded.

Next we show that $(S(t))_{t \geq 0}$ is not a compact semigroup. We use the compactness criterion from [12, Problem IV.13.3] that a subset $K$ of $l_{1}$ is strongly relatively compact (and thus weakly sequentially relatively compact) if and only if it is bounded and $\lim _{n \rightarrow \infty} \sum_{i=n}^{\infty}\left|a_{i}\right|=0$ uniformly for $\mathbf{a}=\left(a_{1}, a_{2}, \ldots\right) \in K$ or, in other words, for any $\epsilon>0$ there is $n$ such that for any $\mathbf{a} \in K$.

$$
\sum_{i=n}^{\infty}\left|a_{i}\right|<\epsilon .
$$

Let us fix some $n>1$ and, for any $m>n$, take $\mathbf{e}_{m}^{\prime}=m^{-1} \mathbf{e}_{m}$ so that $\left\|\mathbf{e}_{m}^{\prime}\right\|_{1}=1$ and $S(t) \mathbf{e}_{m}^{\prime}=m^{-1}\left(s_{1 m}(t), s_{2 m}(t), \ldots, s_{m m}(t), 0, \ldots\right)$. Then, using (26), we can calculate the reminder of the series $\left\|S(t) \mathbf{e}_{m}^{\prime}\right\|_{1}$

$$
\begin{aligned}
R_{n}\left(S(t) \mathbf{e}_{m}^{\prime}\right) & =m^{-1} \sum_{k=n}^{m} k s_{k, m}(t)=m^{-1} \sum_{k=n}^{m} k\left(\begin{array}{c}
m \\
m-k
\end{array}\right) e^{-m t}\left(e^{t}-1\right)^{m-k} \\
& =m^{-1} \sum_{k=n}^{m} k\left(\begin{array}{c}
m \\
k
\end{array}\right) e^{-k t}\left(1-e^{-t}\right)^{m-k} .
\end{aligned}
$$


For an arbitrary fixed $t^{\prime}>0$ we denote $p=e^{-t^{\prime}}$ and $q=1-e^{-t^{\prime}}$ so that $0<p, q<1$ and $p+q=1$. Further, let $\alpha:=p / q$. Then

$$
\begin{aligned}
R_{n}\left(S\left(t^{\prime}\right) \mathbf{e}_{m}^{\prime}\right) & =m^{-1} \sum_{k=n}^{m} k\left(\begin{array}{c}
m \\
k
\end{array}\right) p^{k} q^{m-k}=\alpha q^{m} \sum_{k=n}^{m}\left(\begin{array}{c}
m-1 \\
k-1
\end{array}\right) \alpha^{k-1} \\
& =\alpha q^{m} \sum_{k=n-1}^{m-1}\left(\begin{array}{c}
m-1 \\
k
\end{array}\right) \alpha^{k}=\alpha q^{m}\left((1+\alpha)^{m-1}-\sum_{k=0}^{n-2}\left(\begin{array}{c}
m-1 \\
k
\end{array}\right) \alpha^{k}\right) \\
& =p-\alpha q^{m} \sum_{k=0}^{n-2}\left(\begin{array}{c}
m-1 \\
k
\end{array}\right) \alpha^{k} .
\end{aligned}
$$

Now, for any fixed $n$, the sum $\sum_{k=0}^{n-2}\left(\begin{array}{c}m-1 \\ k\end{array}\right) \alpha^{k}$ is a polynomial in $m$ of degree $n-2$ with fixed coefficients which do not depend on $m$ and therefore, since $q<1$, there is $m_{0}$ such that

$$
\left|\alpha q^{m} \sum_{k=0}^{n-2}\left(\begin{array}{c}
m-1 \\
k
\end{array}\right) \alpha^{k}\right|<p / 2
$$

for all $m \geq m_{0}$. Hence

$$
R_{n}\left(S\left(t^{\prime}\right) \mathbf{e}_{m}^{\prime}\right) \geq p / 2
$$

for $m \geq m_{0}$. Summarizing, considering $K\left(t^{\prime}\right)=e^{t^{\prime} \mathcal{S}}\left\{\mathbf{e}_{1}^{\prime}, \ldots, \mathbf{e}_{n}^{\prime}, \ldots\right\}$, where $\left\|\mathbf{e}_{n}^{\prime}\right\|_{1}=1$, there is an number $p>0$ such that for any $n$ there is $m$, with $R_{n}\left(S\left(t^{\prime}\right) \mathbf{e}_{m}^{\prime}\right)$, satisfying (29). This, however, contradicts (28). Hence, $K\left(t^{\prime}\right)$ is not relatively compact for any $t^{\prime}$ and thus $(S(t))_{t \geq 0}$ is not compact for any $t$.

Representation formulae (26) and (27) allow to gain a more detailed information about this fragmentation process, supporting some claims made in the introduction.

\section{Corollary 1 Consider the fragmentation process introduced in Theorem 3. Then}

(i) $(S(t))_{t \geq 0}$ does not extend continuously to $l^{1}$;

(ii) The operator norm of $S^{[2]}(t)$ satisfies $\left\|S^{[2]}(t)\right\|_{\mathcal{L}\left(l_{1}^{1}\right)}=e^{-t}$ and thus $(S(t))_{t \geq 0}$ has AEG property despite being non-compact.

Proof Let us consider $\mathbf{u}=\left(n^{-2}\right)_{n \geq 1} \in l^{1} \backslash l_{1}^{1}$. Then, using (27),

$$
s_{1, n}(t)=n\left(1-e^{-t}+e^{-t}\left(1-e^{-t}\right)^{n-1}\right),
$$

hence for any $t>0$

$$
\|S(t) \mathbf{u}\|_{0} \geq \sum_{n=1}^{\infty} s_{1, n} u_{n}=\sum_{n=1}^{\infty} n^{-1}\left(1-e^{-t}+e^{-t}\left(1-e^{-t}\right)^{n-1}\right) \geq\left(1-e^{-t}\right) \sum_{n=1}^{\infty} n^{-1}=\infty .
$$

Therefore the operator $S(t)$ does not extend to a bounded operator on $l^{1}$ for any $t>0$.

To prove the second statement, we observe that

$$
\left\|S^{[2]}(t)\right\|_{\mathcal{L}\left(l_{1}^{1}\right)}=\sup _{n \geq 2} \frac{1}{n} \sum_{i=2}^{n} i\left(\begin{array}{l}
n \\
i
\end{array}\right) e^{-i t}\left(1-e^{-t}\right)^{n-i}=e^{-t} \sup _{n \geq 2}\left(1-\left(1-e^{-t}\right)^{n-1}\right)=e^{-t},
$$

hence the statement follows by Lemma 2. 
3.2 Fragmentation with bounded fragmentation rates

As in the previous example, we consider the simplest case, that is, we put $a_{j}=1, j \geq 2$, and $\left(b_{k, n}\right)_{1 \leq k<n, n \geq 2}$ satisfying (24). In this case the system (3) becomes

$$
\begin{aligned}
& u_{1}^{\prime}=2 u_{2}+\sum_{j=3}^{\infty} u_{j}, \\
& u_{i}^{\prime}=-u_{i}+u_{i+1}, \quad i=2, \ldots
\end{aligned}
$$

We note that, contrary to the previous case, the system (30) generates uniformly continuous positive semigroups both in $l^{1}$ and in $l_{1}^{1}$. Since the semigroup in $l_{1}^{1}$ is the restriction of the semigroup in $l^{1}$, we use the symbol $(\mathcal{S}(t))_{t \geq 0}$ to denote the infinite matrix (10) while $\left(S_{0}(t)\right)_{t \geq 0}$ and $(S(t))_{t \geq 0}$ will denote the semigroups defined by this matrix in $l^{1}$ and $l_{1}^{1}$, respectively. Similar convention will be applied to the operators defined by expressions $\mathcal{A}$ and $\mathcal{B}$ [see (7) and (8)], that is, $A_{0}$ and $B_{0}$ define restrictions of $\mathcal{A}$ and $\mathcal{B}$, respectively, to $l^{1}$ whereas $A$ and $B$ are respective operators in $l_{1}^{1}$, as before.

While in $l_{1}^{1}$ the fragmentation semigroup is contractive, as in the unbounded case, its behaviour in $l^{1}$ is different. Indeed, let $\mathbf{u}(t)=S_{0}(t) \stackrel{\circ}{\mathbf{u}}$ with $\stackrel{\circ}{\mathbf{u}} \in l_{+}^{1}$. Since the operators $A_{0}$ and $B_{0}$ are bounded, we can add rows of (30), split summation between the diagonal and off-diagonal entries and factor out the time derivative from the sum on the left hand side, getting

$$
\frac{d}{d t}\|\mathbf{u}(t)\|_{0}=\sum_{j=2}^{\infty} u_{j}(t)=\left\|\mathbf{u}^{[2]}(t)\right\|_{0}
$$

which shows that the number of particles increases.

Before formulating a counterpart of Theorem 3, we observe that both $\left(S_{0}(t)\right)_{t \geq 0}$ and $(S(t))_{t \geq 0}$, being uniformly bounded, are analytic as the sums of uniformly converging power series. Thus, we only can prove the following result.

Theorem 4 The fragmentation semigroups $(S(t))_{t \geq 0}$ and $\left(S_{0}(t)\right)_{t \geq 0}$, associated with the system (30), are not compact in the respective spaces.

Proof As before, we use Lemma 1 to find that $\mathbf{u}(t)=\mathcal{S}(t) \stackrel{\circ}{\mathbf{u}}$ is given by

$$
\begin{aligned}
& s_{1,1}(t)=1, \\
& s_{1, n}(t)=\sum_{i=0}^{n-3}\left(1-e^{-t} \sum_{k=0}^{i} \frac{t^{k}}{k !}\right)+2\left(1-e^{-t} \sum_{k=0}^{n-2} \frac{t^{k}}{k !}\right), \quad n>1 \\
& s_{k, n}(t)=e^{-t} \frac{t^{n-k}}{(n-k) !}, \quad k>1, n \geq k,
\end{aligned}
$$

so that

$$
u_{k}(t)=e^{-t} \sum_{n=k}^{\infty} \stackrel{\circ}{u}_{n} \frac{t^{n-k}}{(n-k) !}, \quad k>1
$$


Taking the Laplace transform (in either space), we find that for the resolvent of $(S(t))_{t \geq 0}$ (or $\left.\left(S_{0}(t)\right)_{t \geq 0}\right)$ we have

$$
(R(\lambda, \mathcal{F}) \stackrel{\circ}{\mathbf{u}})_{k}=\sum_{n=k}^{\infty} \frac{\stackrel{\circ}{u}_{n}}{(\lambda+1)^{n-k+1}}, \quad k \geq 2
$$

If $R(\lambda, F)$, or $R\left(\lambda, F_{0}\right)$, were compact then, by the Fisher-Riesz theory, $\lambda=-1$ would be a finite order pole so that for some fixed $m$ there would exist a finite non-zero limit $\lim _{\lambda \rightarrow-1}(\lambda+1)^{m} R(\lambda, \mathcal{F})$ in the uniform operator topology of the respective space. However, taking any coordinate of $R(\lambda, \mathcal{F})$ with $k \geq 2$ and considering $\mathbf{f}=\left(\delta_{l r}\right)_{l \geq 1}$ with $r>k$ we see that $m$ has to depend on $r$ which shows that $\lambda=-1$ is an essential singularity.

It is interesting to note that, unlike in the unbounded case described in Corollary 1 (ii), here the semigroup $(S(t))_{t \geq 0}$, besides being non-compact, also does not have the AEG property. The reason for this could be that in the previous case the fragmentation rates, which increased with the size of the particles, drive the system faster to the equilibrium, whereas in the present model the constant fragmentation rates are too weak for an exponential rate of decay.

Corollary 2 Consider the fragmentation process as in Theorem 4. Then

$$
\left\|S^{[2]}(t)\right\|_{\mathcal{L}\left(l_{1}^{1}\right)}=1
$$

and thus $(S(t))_{t \geq 0}$ does not have the AEG property.

Proof Using (31) we get

$$
\left\|S^{[2]}(t)\right\|_{\mathcal{L}\left(l_{1}^{1}\right)}=e^{-t} \sup _{l \geq 2} \frac{1}{l} \sum_{j=2}^{l} \frac{j t^{l-j}}{(l-j) !}
$$

and

$$
\begin{aligned}
\frac{1}{l} \sum_{j=2}^{l} \frac{j t^{l-j}}{(l-j) !} & =\frac{1}{l} \sum_{r=0}^{l-2} \frac{(l-r) t^{r}}{r !}=\sum_{r=0}^{l-2} \frac{t^{r}}{r !}-\frac{t}{l} \sum_{r=0}^{l-3} \frac{t^{r}}{r !}=\frac{t^{l-2}}{(l-2) !}+\left(1-\frac{t}{l}\right) \sum_{r=0}^{l-3} \frac{t^{r}}{r !} \\
& =\frac{t^{l-2}}{(l-2) !}+e^{t}\left(1-\frac{t}{l}\right)-\left(1-\frac{t}{l}\right) \sum_{r=l-2}^{\infty} \frac{t^{r}}{r !} \geq e^{t}-\frac{t e^{t}}{l}-\sum_{r=l-2}^{\infty} \frac{t^{r}}{r !},
\end{aligned}
$$

where the last estimate was obtained by discarding two positive terms. Next, the last two terms are monotonically decreasing to 0 and thus

$$
\left\|S^{[2]}(t)\right\|_{\mathcal{L}\left(l_{1}^{1}\right)}=e^{-t} \sup _{l \geq 2} \frac{1}{l} \sum_{j=2}^{l} \frac{j t^{l-j}}{(l-j) !} \geq e^{-t} \sup _{l \geq 2}\left(e^{t}-\frac{t e^{t}}{l}-\sum_{r=l-2}^{\infty} \frac{t^{r}}{r !}\right)=1 .
$$

From the considerations in Section 2 , we see that $\left\|S^{[2]}(t)\right\|_{\mathcal{L}\left(l_{1}^{1}\right)} \leq 1$, hence $\left\|S^{[2]}(t)\right\|_{\mathcal{L}\left(l_{1}^{1}\right)}=1$ and so the type of $\left(S^{[2]}(t)\right)_{t \geq 0}$ is zero. Thus, by Lemma 2, the convergence to the equilibrium state cannot be exponential. 


\section{Chaos in fragmentation models with growth}

As for the pure death problems, discussed in the introduction, it is reasonable to consider fragmentation processes in which the clusters can grow. For instance, in biological interpretations, a cluster of cells can grow by division of the cells forming it, provided some daughter cells remain in the cluster. Another example of such a model are particles immersed in a solute, which grow by the surface deposition of new particles from the solute.

In this section again we consider the fragmentation model in which only a monomer can detach from the parent. Also, we assume constant fragmentation and growth rates. Then the system modelling the evolution can be obtained by adding a diagonal entry to (30), which results in

$$
\begin{aligned}
& u_{1}^{\prime}=c u_{1}+2 d u_{2}+d \sum_{j=3}^{\infty} u_{j}, \\
& u_{j}^{\prime}=c u_{j}-d u_{j}+d u_{j+1}, \quad j \geq 2,
\end{aligned}
$$

where $c$ is the net growth rate and $d$ is the fragmentation rate. Here, unlike in the pure fragmentation case, the dynamics depends on $c$ and $d$, hence we have not set them equal to 1 . Since the diagonal entry is bounded, (33) can be analysed both in $l^{1}$ and in $l_{1}^{1}$. We denote by $\mathcal{G}$ the matrix of the coefficients on the right hand side of (33). Then $G_{0}$ and $\left(T_{0}(t)\right)_{t \geq 0}$ will denote the operator induced by $\mathcal{G}$ and the semigroup generated by it in $l^{1}$, while $G$ and $(T(t))_{t \geq 0}$ are the corresponding operator and the semigroup in $l_{1}^{1}$. Further, we represent the dual space to $l_{1}^{1}$ as

$$
l_{-1}^{\infty}=\left\{\boldsymbol{\phi} ; \sup _{n \geq 1}\left|n^{-1} \phi_{n}\right|<\infty\right\}
$$

so that the duality pairing between $l_{1}^{1}$ and $l_{-1}^{\infty}$ coincides with the standard $l^{1} \times l^{\infty}$ pairing

$$
<\mathbf{u}, \boldsymbol{\phi}>=\sum_{i=1}^{\infty} u_{i} \phi_{i}, \quad \mathbf{u} \in l_{1}^{1}, \boldsymbol{\phi} \in l_{-1}^{\infty} .
$$

Theorem 5 Assume $0<c<2 d$. Then

(a) The semigroup $\left(T_{0}(t)\right)_{t \geq 0}$ is chaotic in $l^{1}$.

(b) The semigroup $(T(t))_{t \geq 0}$ is not chaotic in $l_{1}^{1}$ but it is subchaotic with the maximal chaoticity space

$$
X_{c h}=\left\{\mathbf{u} \in l_{1}^{1} ;<\mathbf{u}, \boldsymbol{\psi}>=0\right\},
$$

where $\boldsymbol{\psi}=(n)_{n \geq 1}$.

Proof To test (33) for a chaotic behaviour, we use the criteria provided in [11, Theorems 3.1 and 3.3]. For this, we consider the eigenproblem for (33)

$$
\begin{aligned}
& \lambda v_{1}=c v_{1}+2 d v_{2}+d \sum_{j=3}^{\infty} v_{j}, \\
& \lambda v_{j}=c v_{j}-d v_{j}+d v_{j+1}, \quad j \geq 2 .
\end{aligned}
$$


We simplify the problem by introducing $\mu=(\lambda-a) / d$ with $a=c-d$ and $\mathbf{g}(\mu)=\mathbf{v}(\lambda)$; this yields

$$
\begin{aligned}
& \mu g_{1}=g_{1}+2 g_{2}+\sum_{j=3}^{\infty} g_{j}, \\
& \mu g_{j}=g_{j+1}, \quad j \geq 2 .
\end{aligned}
$$

We observe that for $j \geq 2$ the system is just the eigenvalue system for the pure death system with proliferation with the solution $g_{j}=\mu^{j-2} g_{2}$ for $j \geq 3$. However, $g_{2}$ is coupled with all other components through the first equation in (35), that is, we have

$$
(\mu-1) g_{1}=2 g_{2}+g_{2} \sum_{j=3}^{\infty} \mu^{j-2}=g_{2} \frac{2-\mu}{1-\mu},
$$

provided $|\mu|<1$. We can specify either $g_{1}$ or $g_{2}$. The better choice is to set $g_{2}=1$ in which case $g_{1}(\mu)=(\mu-2)(\mu-1)^{-2}$. It is clear that the selection

$$
\mu \rightarrow \mathbf{g}(\mu)=\left(g_{1}(\mu), 1, \mu, \ldots, \mu^{n}, \ldots\right)
$$

is both $l^{1}-$ and $l_{1}^{1}$-analytic in the unit disc $|\mu|<1$. Further, $\{\lambda \in \mathbb{C} ; \lambda=c-d+d \mu,|\mu|<$ $1\} \cap i \mathbb{R} \neq \varnothing$ if and only if $0<c<2 d$. Using the power series expansion

$$
\frac{\mu-2}{(\mu-1)^{2}}=-\sum_{n=0}^{\infty}(n+2) \mu^{n}
$$

we can write

$$
\mathbf{g}(\mu)=\sum_{n=0}^{\infty} \mathbf{g}_{n} \mu^{n}, \quad|\mu|<1,
$$

where $\mathbf{g}_{n}=\left(g_{i n}\right)_{i \geq 1}, n \geq 0$, are defined by

$$
g_{i n}= \begin{cases}-(n+2) & \text { for } i=1 \\ 0 & \text { for } 1<i<n+2 \text { and } i>n+2, \\ 1 & \text { for } i=n+2\end{cases}
$$

By [7, Theorem 1], to prove that (33) generates chaotic dynamics in $l^{1}$ (respectively in $l_{1}^{1}$ ) it is enough to show that the only solution $\phi=\left(\phi_{n}\right)_{n \geq 1} \in l^{\infty}$ (respectively, $l_{-1}^{\infty}$ ) of

$$
g_{1 n} \phi_{1}+g_{2 n} \phi_{2}+\cdots=0, \quad n \geq 0,
$$

is $\phi=(0,0, \ldots)$. In our case the system (36) takes a particularly easy form

$$
-(n+2) \phi_{1}+\phi_{n+2}=0, \quad n \geq 0,
$$

which has one, up to a constant multiplier, nontrivial solution $\psi=(n+1)_{n \geq 0}=(n)_{n \geq 1}$. It is clear that $\psi \notin l^{\infty}$, hence $\left(T_{0}(t)\right)_{t \geq 0}$ is a chaotic semigroup in $l^{1}$. On the other hand, $\psi \in l_{-1}^{\infty}$ and [11, Theorem 3.1] is not sufficient to fully determine the dynamics of $(T(t))_{t \geq 0}$. However, using [5, Criterion 3.3, Lemma 3.4], we can conclude that $(T(t))_{t \geq 0}$ is chaotic in the subspace $X_{c h}$ defined by (33).

This result does not prevent $(T(t))_{t \geq 0}$ to be chaotic in the whole space. To rule out this possibility, we look at the adjoint problem. Since finite sequences are dense in $l_{1}^{1}$, it is clear that the adjoint $G^{*}$ to $G$ is given by the matrix transposed to $\mathcal{G}$. Then, by operations analogous 
to those leading to (35), we reduce seeking eigenvalues and eigenvectors of $G^{*}$ to solving the system

$$
\begin{aligned}
\phi_{1} & =\lambda \phi_{1}, \quad 2 \phi_{1}=\lambda \phi_{2}, \\
\phi_{1}+\phi_{n-1} & =\lambda \phi_{n}, \quad n \geq 3 .
\end{aligned}
$$

We have the following possibilities. If $\lambda=0$ and $\phi_{1}=0$ then, skipping the second equation, we obtain $\phi_{n}=-\phi_{1}=0$ for all $n \geq 2$. If $\lambda \neq 0$ and $\phi_{1}=0$, then $\phi_{2}=0$ from the second equation and then successively $\phi_{n}=0$ for all $n \geq 3$. Finally, if $\lambda \neq 0$ and $\phi_{1} \neq 0$, then $\lambda=1$. Setting $\phi_{1}=1$, we get $\phi_{2}=2$ and $\phi_{n}=\phi_{n-1}+1$ which is an arithmetic progression giving $\phi_{n}=n$. Since $\phi=(n)_{n \geq 1} \in l_{-1}^{\infty}$, the point spectrum of $G^{*}$ is not empty and therefore $(G(t))_{t \geq 0}$ is not hypercyclic (and thus not chaotic) in $l_{1}^{1}$, by [11, Theorem 3.3]. However, using [5, Theorem 4.1] we may state that $(G(t))_{t \geq 0}$ may have a dense orbit at most in the subspace of $l_{1}^{1}$ annihilating $(n)_{n \geq 1}$, which is consistent with the previous part of the proof.

Acknowledgments The author thanks the anonymous referees whose careful reading of the manuscript contributed to a significant improvement of the final version.

Open Access This article is distributed under the terms of the Creative Commons Attribution Noncommercial License which permits any noncommercial use, distribution, and reproduction in any medium, provided the original author(s) and source are credited.

\section{References}

1. Abramowitz, M., Stegun, I.A.: Handbook of Mathematical Functions. National Bureau of Standards, Washington (1972)

2. Ball, J.M., Carr, J.: The discrete coagulation-fragmentation equations: existence, uniqueness and density conservation. J. Stat. Phys. 61, 203-234 (1990)

3. Banasiak, J., Lachowicz, M.: Topological chaos for birth-and-death-type models with proliferation. Math. Models Methods Appl. Sci. 12(6), 755-775 (2002)

4. Banasiak, J., Arlotti, L.: Perturbations of positive semigroups with applications. Springer, London (2006)

5. Banasiak, J., Moszyński, M.: A generalization of Desch-Schappacher-Webb criteria for chaos. Discr. Cont. Dyn. Sys. A 12(5), 959-972 (2005)

6. Banasiak, J.: Positivity in natural sciences. In: Capasso, V., Lachowicz, M. (eds.) Multiscale Problems in the Life Sciences, LNM 1940, pp. 1-89. Springer, Berlin (2008)

7. Banasiak, J.: Chaos in Kolmogorov systems with proliferation-general criteria and applications. J. Math. Anal. Appl. (2011, in press). doi:10.1016/j.jmaa.2010.12.054

8. Banasiak, J., Lamb, W., Langer, M.: Long time behaviour and asymptotics of solutions to discrete fragmentation equation (work in progress)

9. Carr, J., da Costa, F.P.: Asymptotic behaviour of solutions to the coagulation-fragmentation equations. II. Weak fragmentation. J. Stat. Phys. 77(1-2), 89-123 (1994)

10. Clément, Ph., Heijmans, H.J.A.M., Angenent, S.C., van Duijn, J., de Pagter, B.: One-Parameter Semigroups. North Holland, Amsterdam (1987)

11. Desch, W., Schappacher, W., Webb, G.F.: Hypercyclic and chaotic semigroups of linear operators. Ergodic Theory Dyn. Syst. 17, 793-819 (1997)

12. Dunford, N., Schwartz, J.T.: Linear Operators, Part I: General Theory. Wiley, New York (1988)

13. Engel, K.-J., Nagel, R.: A Short Course on Operator Semigroups. Springer, New York (2006)

14. Grosse-Erdmann, K.-G., Peris, A.: Linear Chaos. Springer, Berlin (in press) (2011)

15. Martcheva, M., Thieme, H.R.: Infinite ODE Systems Modelling Size-Structured Metapopulations, Macroparasitic Diseases and Prion Proliferation, In: Magal, P., Ruan, S. (eds.), Structured Population Models in Biology and Epidemiology, LMN 1936. Springer, Berlin (2008)

16. Protopopescu, V., Azmy, Y.Y.: Topological chaos for a class of linear models. Math. Models Meth. Appl. Sci. 2(1), 79-90 (1992) 